\title{
Nasal carriage of Staphylococcus aureus and presence of genetically identical strains on the mobile phones carried by healthcare providers in the intensive care unit.
}

\author{
Nevcivan Guldas ${ }^{1}$, Ayse Nur Sari², and Zeynep GULAY ${ }^{3}$ \\ ${ }^{1}$ Dokuz Eylul University Faculty of Medicine \\ ${ }^{2}$ Dokuz Eylul Universitesi \\ ${ }^{3}$ Dokuz Eylul University School of Medicine
}

June 2, 2020

\begin{abstract}
Aims of the study: Staphylococcus aureus colonized in the nose of healthcare providers is an important risk factor for the development of hospital-acquired staphylococcal infections. Cross-contamination of this bacterium between the hands of healthcare providers and the surfaces is known. In this study, we aimed to evaluate the clonal relationship between eight Staphylococcus aureus strains isolated from the nose of healthcare providers and five Staphylococcus aureus strains isolated from mobile phones carried by healthcare providers. Methods: The clonal relationship between the strains and molecular epidemiological status were investigated by the pulsed-field gel electrophoresis method. Results: The first and third strains are isolated from the mobile phone and the nose of a healthcare provider working in the intensive care unit were the same. The second and fourth strains were isolated from the mobile phone and nose of another healthcare provider working in the intensive care unit were the same. The fifth strain, which was found to be the same as the second and fourth strains, was isolated from the mobile phone of another healthcare provider working in the intensive care unit. No similarity was observed between the other strains. Conclusion: Our findings indicate that Staphylococcus aureus strains colonized in the nose of healthcare providers are also transmitted to other surfaces and that the hospital environment and co-used devices pose a risk for spread. For this reason, training of healthcare providers on the infection control procedure, hand hygiene, environmental disinfection and regular cleaning of mobile phones are important components in order to prevent hospital-acquired infections.
\end{abstract}

\section{INTRODUCTION}

Staphylococcus aureus, one of the most commonly isolated agents in both community and hospital-acquired infections worldwide ${ }^{1}$. One of the important risk factors for the development of hospital-acquired staphylococcal infections is $S$. aureus, which is colonized in the noses of healthcare providers ${ }^{2}$. This bacterium can be transmitted to the mobile phones used by healthcare providers through their hands ${ }^{3,4}$.

The relationship between $S$. aureus colonized in the noses of healthcare providers and $S$. aureus detected in their mobile phones is still unclear. The question of "Are mobile phones randomly contaminated with S. aureus or contaminated with $S$. aureuscolonized in the noses of healthcare providers?" is waiting to be answered.

The aim of this study was to evaluate the clonal relationship between $S$. aureus strains isolated from the noses of healthcare providers working in the internal medicine intensive care unit (IMICU) and the operating room, and $S$. aureus strains isolated from the mobile phones of IMICU workers.

\section{METHODS}


During the period from December 2014-January 2015, 96 mobile phones of healthcare providers in IMICU, anesthesia intensive care unit (AICU) and operating room of our hospital were sampled for bacterial contamination. On the other hand, during the same period we investigated nasal $S$. aureus colonization of same healthcare providers. The samples were screened for bacterial pathogens by standart bacteriological procedures. The methicillin sensitivity of the identified $S$. aureus isolates were tested. Eight methicillin-sensitive S.aureus (MSSA) strains isolated from the noses of the healthcare providers and five MSSAstrains isolated from their mobile phones were included in this study. The section from which the strains were isolated, occupational group and sample distribution are presented in

\section{Table I.}

The clonal relationship between the strains and molecular epidemiological status were investigated by the pulsed-field gel electrophoresis (PFGE) method. The bacterial DNA was obtained from each strain by the acromopeptidase method. The DNA macrorestriction analysis was performed according to the protocol prepared by the Scottish MRSA Reference Laboratory with the Sma I restriction method suggested by Bannerman et al. ${ }^{5}$ The Chef DR III (Biorad) device was used for PFGE, and EMRSA 2 and EMRSA 6 strains were included in the study with the aim of making a comparison. After electrophoresis, the gel was stained with ethidium bromide $(0.5 \mu \mathrm{g} / \mathrm{ml})$, and DNA bands were observed. The clonal relationship between the strains was evaluated according to the criteria described by Tenover et al. from the DNA band patterns formed in the gel after PFGE ${ }^{6}$.

\section{RESULTS}

In the evaluation of the strains with the PFGE analysis, the 1st and 3 rd strains, and the $2 \mathrm{nd}, 4$ th and 5 th strains were identical. The 1st strain was isolated from the mobile phone of a healthcare provider working in the IMICU, and the $3 \mathrm{rd}$ strain was isolated from the nose of the same worker. Similarly, the 2 nd strain was isolated from the mobile phone of a healthcare provider working in the IMICU, and the 4 th strain was isolated from the nose of the same worker. The 5 th strain, which was observed to be identical with the 2 nd and 4 th strains, was isolated from the mobile phone of another healthcare provider working in the IMICU. No similarity was observed between other strains ( Figure 1) .

\section{DISCUSSION}

Bacterial contamination of healthcare providers' mobile phones is an extensively studied subject ${ }^{7}$. However, the relationship between nasal colonization and mobile phone contamination is not well understood. In this study, we conducted PFGE to investigate the closeness between S.aureus strains isolated from the noses and mobile phones of healthcare providers. We found that out $S$. aureus strains isolated from the mobile phones of two healthcare providers and $S$. aureus strains isolated from their noses were genetically identical. This shows that bacteria colonized in the nose contaminate mobile phones. Kanayama et al. showed that $S$. aureusstrains isolated from the mobile phones of nurses and $S$. aureusstrains detected in their hands were genetically identical and mentioned the cross-contamination between mobile phones and hands ${ }^{8}$. Furthermore, it was found out in our study that the strain isolated from the nose and mobile phone of a healthcare provider and the strain detected on the mobile phone of another healthcare provider were identical, which shows that $S$.aureus strains colonized in the noses of healthcare providers can be transmitted to other surfaces through hands, and that the hospital environment and the shared devices pose a risk for spread. Similarly, S. aureusstrains, which were also genetically identical in the study of Kanayama et al. were observed in many mobile phones and the hands of people using them, indicating that these strains spread to large areas within the hospital through the hands of healthcare providers using contaminated mobile phones ${ }^{8}$.

In the study of Chang et al. which examines the bacterial contamination of mobile phones of healthcare providers working in the operating room and compares this with the hand and nose colonization of the same healthcare providers, it was found out that the rate of mobile phone contamination was $98 \%$, and also the bacteria in the mobile phones, hands, and noses of the healthcare providers were identical at the rate of 94.3 $\%^{9}$ 
In their study, Brady et al. demonstrated the relationship between nasalS. aureus carriage of healthcare providers and the $S$. aureus contamination of the mobile phones used by patients and emphasized the risk of cross-contamination ${ }^{10}$. Shi et al. reported the cross-contamination of $S$. aureus on the surfaces which healthcare providers contacted with their hands in a large city hospital ${ }^{11}$.

In many studies conducted, it was reported that nasal $S$. aureuscarriage was detected at a high rate in healthcare providers. Chang et al. detected that S. aureus nasal carriage in 19,9\% of healthcare providers 9. According to other studies in the literature, this rate was reported to be $30 \%$ in a study conducted in Argentina, $12 \%$ in a study conducted in Ethiopia, and $26 \%$ in a study conducted in India ${ }^{12-14}$.

The findings of our study indicate that mobile phones used by healthcare providers may be reservoirs for potential pathogens in nasal colonization. The most common potential pathogen $S$. aureus is encountered in surgical site infections, pneumonia, septicemia and instrument-related infections. The identification of the relationship between $S$. aureus strains isolated from these infections and $S$. aureus strains isolated from the noses and mobile phones of healthcare providers will be useful in determining the ways of infection transmission.

\section{CONCLUSION}

In the light of these findings, it is important that mobile phone use should be regulated and cleanliness should not be neglected in areas with a high risk of infection in the hospital. Hand hygiene is one of the basic factors in the prevention of hospital infections ${ }^{15}$. Hand hygiene must be definitely ensured after the use of a mobile phone and before contact with the patient. In addition to appropriate hand hygiene, it is important to determine the healthcare providers with $S$. aureus nasal carriage, to treat the carriage and to pay attention to the use of shared devices to prevent hospital-acquired infections.

\section{AUTHOR CONTRIBUTIONS :}

All authors were involved in the conception or design of the study, data analysis and interpretation, drafting, critical revision and approval of article.

Table I: Isolated section, occupational group and sample distribution of the strains

\begin{tabular}{lllll}
\hline Strain No & Sample used in isolation & Section & Occupational group & PFGE Pattern \\
\hline 1 & Mobile phone & IMICUA & Doctor 1 & A \\
2 & Mobile phone & IMICU & Doctor 2 & B \\
3 & Nose & IMICU & Doctor 1 & A \\
4 & Nose & IMICU & Doctor 2 & B \\
5 & Mobile phone & IMICU & Doctor 3 & B \\
6 & Mobile phone & IMICU & Allied Health Personnel 1 & C \\
7 & Mobile phone & IMICU & Nurse 1 & D \\
8 & Nose & IMICU & Doctor 4 & E \\
9 & Nose & IMICU & Allied Health Personnel 2 & F \\
10 & Nose & Operating Room & Allied Health Personnel 3 & G \\
11 & Nose & IMICU & Doctor 5 & H \\
12 & Nose & Operating Room & Allied Health Personnel 4 & I \\
13 & Nose & IMICU & Allied Health Personnel 5 & J \\
\hline
\end{tabular}

Anternal medicine intensive care unit

Figure 1: PFGE band patterns of strains (Batches 1-13: strains isolated from the noses and mobile phones of healthcare workers, K1: EMRSA 2, K2: EMRSA 6) 


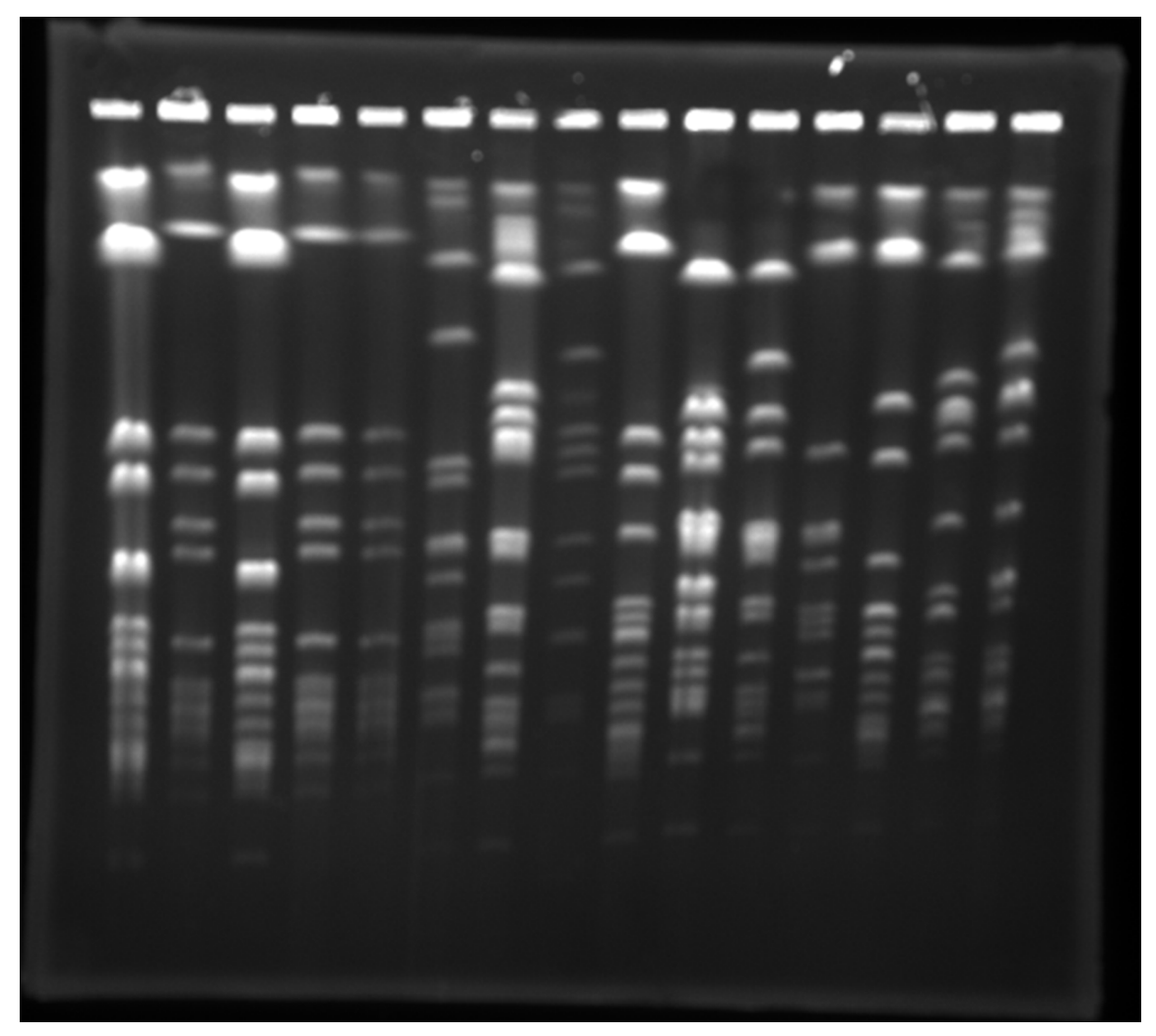

\section{REFERENCES}

1. Tong SYC, Davis JS, Eichenberger E, Holland TL, Fowler VG Jr. Staphylococcus aureus infections: epidemiology, pathophysiology, clinical manifestations, and management. Clin Microbiol Rev. 2015;28:603-661.

2. Chiang FY, Climo M. Staphylococcus aureus Carriage and Health Care-acquired Infection. Curr Infect Dis Rep 2002; 4:498-504.

3. Brady RR, Wasson A, Stirling I, McAllister C, Damani NN. Is your phone bugged? The incidence of bacteria known to cause nosocomial infection on healthcare workers' mobile phones. J Hosp Infect $2006 ; 62: 123-125$.

4. Khivsara A, Dahashree B. Typing of Staphylococcus aureus from mobile phones and clinical samples. Curr Sci 2006; 90: 910-912.

5. Bannerman TL, Hancock GA, Tenover FC, Miller JM. Pulsed-field gel electrophoresis as a replacement for bacteriophage typing of Staphylococcus aureus. J Clin Microbiol 1995; 33: 551-555.

6. Tenover FC, Arbeit RD, Goering RV, et al. Interpreting chromosomal DNA restriction patterns produced by pulsed-field gel electrophoresis: criteria for bacterial strain typing. J Clin Microbiol 1995; 33: 2233-2239.

7. Haun N, Hooper-Lane C, Safdar N. Healthcare Personnel Attire and Devices as Fomites: A Systematic Review. Infect Control Hosp Epidemiol 2016; 37: 1367-1373.

8. Kanayama A, Takahashi H, Yoshizawa S, Tateda K, Kaneko A, Kobayashi I. Staphylococcus aureus surface contamination of mobile phones and presence of genetically identical strains on the hands of nursing personnel. Am J Infect Control 2017; 45(8): 929-931.

9. Chang CH, Chen SY, Lu JJ, Chang CJ, Chang Y, Hsieh PH. Nasal colonization and bacterial contamination of mobile phones carried by medical staff in the operating room. PloS One 2017; 31;12: e0175811.

10. Brady RR, Hunt AC, Visvanathan A, et al. Mobile phone technology and hospitalized patients: a 
cross-sectional surveillance study of bacterial colonization, and patient opinions and behaviours. Clin Microbiol Infect 2011; 17: 830-835.

11. Shi LS, Xu CJ, Jia HB, Chen W, Zhou XF, Li XH. Spread ofStaphylococcus aureus between medical staff and high-frequency contact surfaces in a large metropolitan hospital. Int J Nurs Sci 2015; 2: 366 -370 .

12. Boncompain CA, Suárez CA, Morbidoni HR. Staphylococcus aureusnasal carriage in health care workers: First report from a major public hospital in Argentina. Rev Argent Microbiol 2017; 49 : 125-131.

13. Legese H, Kahsay AG, Kahsay A, et al. Nasal carriage, risk factors and antimicrobial susceptibility pattern of methicillin resistant Staphylococcus aureus among healthcare workers in Adigrat and Wukro hospitals, Tigray, Northern Ethiopia. BMC Res Notes 2018; 11: 250.

14. Srivalli B, Ekyshwarya S, Setty CR. Nasal carriage of methicillin resistant Staphylococcus aureus (MRSA) among health care workers in a tertiary care hospital. Int J Biol Med Res 2018; 9: 6348-6351.

15. Song X, Stockwell DC, Floyd T, Short BL, Singh N. Improving hand hygiene compliance in health care workers: strategies and impact on patient outcomes. Am J Infect Control 2013; 41 : e101-105. 